This is the final peer-reviewed accepted manuscript of:

S. Nesci (2020). The mitochondrial permeability transition pore in cell death: $A$ promising drug binding bioarchitecture. Medicinal Research Reviews, 40, 811-817.

The final published version is available online at: https://doi.org/10.1002/med.21635

Rights / License:

The terms and conditions for the reuse of this version of the manuscript are specified in the publishing policy. For all terms of use and more information see the publisher's website. 


\title{
The mitochondrial permeability transition pore in cell death: a promising drug binding bio-architecture
}

\author{
Salvatore Nesci \\ Department of Veterinary Medical Sciences, University of Bologna \\ Via Tolara di Sopra, 5040064 - Ozzano Emilia (BO), ITALY \\ salvatore.nesci@unibo.it
}

\begin{abstract}
Bioenergetic failure often features programmed cell death involved in some severe pathologies. When the cell is fated to die, the inner mitochondrial membrane becomes permeable to ions and solutes, due to the formation and opening of a channel known as mitochondrial permeability transition pore (mPTP). Up to now, the still mPTP elusive structure and mechanism prevented any attempt to identify/design drugs to rule its formation and limit cell death. Latest advances, which strongly suggest that the $F_{1} F_{0}$-ATPase can coincide with the MPTP, open new perspectives in therapy. Compounds targeting and inhibiting cyclophilin $D$, a known mPTP promoter, could be exploited to block mPTP formation. Moreover, if the mPTP- $\mathrm{F}_{1} \mathrm{~F}_{0}-\mathrm{ATPase}$ connection will be consolidated, selected $F_{1} F_{0}$-ATPase inhibitors could represent novel therapeutic options to attenuate mPTP-related diseases by directly acting on the molecular mechanism of the mPTP. This intriguing perspective, which raises new hopes to counteract mPTP-related diseases, stimulates further studies to clarify the MPTP architecture and mechanism.
\end{abstract}

Keywords

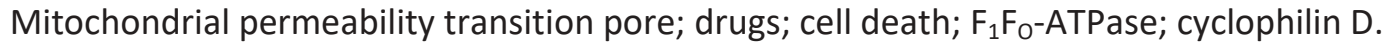




\section{Main text}

A number of human diseases associated with abnormal energy metabolism arise from mitochondrial dysfunctions, which eventually may lead to cell death. In regulated cell death, the pore formation in the inner mitochondrial membrane (IMM) during the mitochondrial permeability transition (mPT) is a common feature, but it remains an enigmatic phenomenon which would involve the formation of pores in the IMM. The "pore" responsible for the lethal cascade of events has been defined as mitochondrial permeability transition pore (mPTP), which would be directly responsible for the bioenergetic impairment. Among the different types of regulated cell death, which differ from each other morphologically, mechanistically and functionally, the mPT-driven necrosis is started by perturbations of the intracellular microenvironment, i.e. severe oxidative stress and cytosolic $\mathrm{Ca}^{2+}$ overload, which results from mPTP opening which in turn depend on cyclophilin D (CyPD) ${ }^{1,2}$. Various insults and stresses can produce a $\mathrm{Ca}^{2+}$ cycle in which a selective $\mathrm{Ca}^{2+}$ efflux from $\mathrm{Ca}^{2+}-$ loaded mitochondria took place before mitochondrial swelling. Moreover, pro-apoptotic stimuli are required to promote intrinsic apoptosis by $\mathrm{Cyt}-\mathrm{C}$ release and consequent caspase activation. Therefore, the mPTP could have a prominent role in regulated cell death and constitute the point of no return in the lethal pathway. The MPTP opening/closing dysregulation is a common element in a wide variety of human disorders in which the excessive MPTP opening induces tissue damage or, on the contrary, the mPTP closing limits apoptosis. The mPTP opening causes mitochondrial dysfunctions by abolishing the transmembrane $\mathrm{H}^{+}$ gradient and preventing ATP synthesis. Accordingly, mitochondrial disorders involved in cellular demise mainly show these features ${ }^{1}$. On theses bases, the potential treatment of diseases featured by increased propensity to MPTP opening encourages to address drug design to modulate MPTP activity.

The molecular architecture of the MPTP is still unresolved, being the molecular components a matter of debate, but an overall consensus exists on the involvement of membrane-bound proteins. The initial belief that the MPTP forms at the IMM and outer mitochondrial membrane (OMM) adjoint sites. The MPTP identity was intensively searched for among the adenine nucleotide translocase (ANT), the mitochondrial phosphate carrier (PiC) and the spastic paraplegia 7 (SPG7). Then the ATP synthasome, an hetero-oligomeric membrane protein complex composed of ANT, PiC and $\mathrm{F}_{1} \mathrm{~F}_{\mathrm{O}}$-ATPase, was also involved ${ }^{3}$. In addition, the OMM components, the voltage-dependent anion channel (VDAC), the translocator protein (TSPO) and BCL-2 family members have been suggested as candidates in mPTP formation ${ }^{1}$. However, the MPTP is primarily an IMM event, while the OMM may play a role in its modulation. Several reports based on genetic manipulations, electrophysiological measurements and mutagenesis of specific aminoacid residues strongly suggest the mitochondrial $F_{1} F_{0}$-ATPase as the main channel-forming component of the mPTP ${ }^{4,5}$.

The $\mathrm{F}_{1} \mathrm{~F}_{\mathrm{O}}$-ATPase is a bi-functional enzyme complex composed by two sector (Fig. 1). A membrane-embedded $\mathrm{F}_{\mathrm{O}}$ sector formed from the subunits $a$, the $c_{\mathrm{n}}$-ring, two membrane-inserted $\alpha$-helices of $b$ subunits and supernumeraries subunits $e, f, g, \mathrm{~A} 6 \mathrm{~L}$, DAPIT and $6.8 \mathrm{kDa}$ proteolipid, which allows $\mathrm{H}^{+}$flow across the inner mitochondrial membrane (IMM). A soluble catalytic $F_{1}$ sector consisting of $(\alpha \beta)_{3}, \gamma, \delta$ and $\varepsilon$ subunits which protrudes in the matrix and synthesizes/hydrolyzes ATP. The two sectors are functionally and structurally connected by a central stalk and a peripheral stalk (formed by the OSCP, $d$, F6 and the extrinsic $\alpha$-helices of $b$ subunits), so as to allow the protonmotive force to drive ATP synthesis; vice versa ATP hydrolysis is coupled to $\mathrm{H}^{+}$pumping from the matrix to the intermembrane space, which polarizes the IMM. The $\mathrm{F}_{1} \mathrm{~F}_{0}-\mathrm{ATPases}$ form supra-molecular dimeric complexes in the IMM by making closer their transmembrane $F_{O}$ domains. The dimeric arrangement contributes to the mitochondrial morphology and to the energy-transduction bioenergetics mechanism. Interestingly, according to an intriguing model, the $F_{1} F_{0}$-ATPase known role of "enzyme of life" that produces ATP turns into the opposite task of "enzyme of death" as energy-dissipating mechanism when the mitochondrial $\mathrm{Ca}^{2+}$ concentration abruptly increases under pathological conditions by forming and opening of $\mathrm{mPTP}^{4}$. Even if the catalytic properties of substrate binding to enzyme are independent of the cation that acts as cofactor, the catalytic sites may adopt different conformations if the natural cofactor $\mathrm{Mg}^{2+}$ is replaced with $\mathrm{Ca}^{2+}$. Recent findings strongly hint that in the latter case the $\mathrm{F}_{1} \mathrm{~F}_{\mathrm{O}^{-}}$ 
ATPase is directly involved in the mPTP formation ${ }^{6}$. Molecular dynamics simulations of the $(\alpha \beta)_{3}$-OSCP complex in the $\mathrm{F}_{1}$ domain strongly suggest that $\mathrm{Ca}^{2+}$ insertion in the enzyme catalytic sites when $\mathrm{Ca}^{2+}$ replaces $\mathrm{Mg}^{2+}$ triggers conformational changes ${ }^{7}$, which are mechanically transmitted from the hydrophilic sector $\mathrm{F}_{1}$ to the membrane portion $F_{O}$ in the IMM. So, the MPTP arises from the dimer dissociation, namely from the detachment of the two membrane $F_{O}$ domains and forms at their interface, probably changing the curvature of the edge of the cristae in the IMM ${ }^{4,8}$. Accordingly, the dimer disassembly during mPTP opening reduces the IMM convexity, thus causing the retraction of the cristae and modifying the mitochondrial morphology. However, the " $c$-ring hypothesis" sustains that the mechanism for channel formation lies within the $c$-ring of the $\mathrm{F}_{\mathrm{O}}$ sector $^{9}$. Recently, $\mathrm{c}$ subunit-knockout cells, the larger cyclosporine A (CsA)-dependent current was not detected, but a detected lower conductance channel was shown to be inhibited by ADP and bongkrekic acid, probably due to the activation of the ANT channel ${ }^{10}$. The hypothesis that the mPTP could be linked to the $\mathrm{F}_{1} \mathrm{~F}_{\mathrm{O}}$-ATPase $c$-ring was substantiated by the detection of serum levels of $c$ subunits with surrogate markers of myocardial ischemia-reperfusion injury ${ }^{11}$ when MPTP opening induces heart damage. In addition, the $\alpha$ synuclein aggregate form interacts with the $F_{1} F_{0}$-ATPase and induce MPTP opening ${ }^{12}$ and may contribute as molecular event to Parkinson's disease.

It is clear that the treatment of MPTP-related diseases may be greatly improved by the identification of drugs that rule the MPTP. Working in this direction, known molecules able to modulate the MPTP immediately raised a great interest. The MPTP activity is known to depend on CyPD, a protein modulator of MPTP opening, and to be inhibited by CsA. Even if CsA effects are routinely related to the mPTP activity, all mammalian cyclophilins are CsA susceptive. Unfortunately, CsA has limited solubility in water, poor bioavailability and a low blood-brain barrier permeability, all features which strongly limit its exploitation in therapy. Recent advances suggest that CyPD binds to the OSCP subunit and interacts with the N-terminal part of $b$ subunit of the $\mathrm{F}_{1} \mathrm{~F}_{\mathrm{O}}$-ATPase ${ }^{4,13}$. Both OSCP and $b$ subunits are part of the peripheral stalk of enzyme. CsA, probably by targeting CyPD can inhibit the $\mathrm{Ca}^{2+}$-activated $\mathrm{F}_{1} \mathrm{~F}_{0}$-ATPase at increasing $\mathrm{Ca}^{2+}$ concentrations without affecting the $\mathrm{Mg}^{2+}$-activated $\mathrm{F}_{1} \mathrm{~F}_{0}-\mathrm{ATPase}^{14}$. Noteworthy, high $\mathrm{Ca}^{2+}$ concentrations and oxidative stress in mitochondria are known to open the mPTP. On these bases, the elucidation of the MPTP structure, whose channel formation mechanism most likely is based on the multi-subunit architecture $F_{1} F_{0}-A T P a s e(s)$, will help to explore pharmacological strategies in drug design and discovery to identify novel, clinically useful mPTP inhibitors. Drugs acting as pore shutters, by targeting the MPTP and preventing the cell death pathway, may have a prominent role in many neurodegenerative diseases. Noteworthy, it is universally accepted that CyPD, without being an essential component of $\mathrm{MPTP}$, is a protein which modulates mPTP formation and opening ${ }^{15}$. However, the drugs for brain diseases are a great challenge due to the blood-brain barrier which is only permeable to lipid-soluble small molecules. The MPTP inhibitors fall in two categories: (i) molecules that target CyPD; (ii) compounds that act as mPTP inhibitors via CyPD-independent mechanism. Up to now, CsA is the best characterized immunosuppressive peptide that binds CypD and inhibits the MPTP. However, other promising compounds are emerging. The macrocyclic natural product, as well as macrocyclic CsA-derivatives and other small molecules can bind the CypD and inhibit the mPTP. The non-immunosuppressive CsA derivatives such as NIM811 and Debio-025 are small molecules which can displace CyPD from its site and desensitize the $\mathrm{mPTP}^{16}$. Sanglifehrin A (SfA), like CSA, is a macrocyclic natural inhibitor of MPTP. SfA inhibits the peptidyl-prolyl cis-trans isomerase activity of CyPD and blocks the pore formation at different site from CsA. The sigmoidal dose-response curve suggests a cooperative effect on MPTP structure ${ }^{17}$. The CyPD is a positive MPTP regulator: the occurrence of CSA or genetic CyPD ablation desensitize the MPTP by increasing the $\mathrm{Ca}^{2+}$ threshold required to activate the MPTP, whose opening is still allowed. The CyPD binding site on the peripheral stalk could have a prominent role in the mechanical signal transduction from the $F_{1}$ to the $F_{0}$ sector where the pore forms $s^{4,7}$. The selective deletion of $b$ subunit or of OSCP subunit in cell lines maintains the MPTP features, even if the loss of these subunits of the peripheral stalk changes the solute permeation by affecting the mPTP size, which becomes smaller. Apart from CsA and CsA derivatives, other compounds such as cinnamic anilides, isoxazoles and benzamides delay mPTP opening and are not cytotoxic at effective 
concentrations ${ }^{16}$. Indeed, "CyPD-independent drugs", directly targeting the MPTP, could act on the $\mathrm{Ca}^{2+}$ dependent mechanism of mPTP formation involving the $F_{1} F_{0}$-ATPase. Interestingly, the $F_{1} F_{0}$-ATPase is a bifunctional enzyme whose ATP synthesis/hydrolysis activities are is only supported in the presence of the natural cofactor $\mathrm{Mg}^{2+}$ bound in the catalytic sites. Conversely, in presence of $\mathrm{Ca}^{2+}$ the $\mathrm{Ca}^{2+}$-activated $\mathrm{F}_{1} \mathrm{~F}_{0^{-}}$ ATPase supports ATP hydrolysis but not ATP synthesis and this feature could be related to triggering mPTP opening (Fig. 2). The development of "CyPD-independent drugs" to block the MPTP formation is extremely attractive, but the main question that remains to be solved is to design or find inhibitors that specifically target the $\mathrm{Ca}^{2+}$-activated $\mathrm{F}_{1} \mathrm{~F}_{\mathrm{O}^{-}}$-ATPase without affecting the physiological bi-functional activity of the $\mathrm{Mg}^{2+}$ activated $\mathrm{F}_{1} \mathrm{~F}_{0}$-ATPase, i.e. ATP synthesis and hydrolysis and likewise have no off-target effects outside the mitochondrial enzyme. The known inhibitors of $F_{O}$ domain of enzyme (oligomycin and DCCD) by blocking the $\mathrm{H}^{+}$translocation inhibit the $\mathrm{Ca}^{2+}$ and $\mathrm{Mg}^{2+}$-activated $\mathrm{F}_{1} \mathrm{~F}_{\mathrm{O}^{-}}$-ATPase, respectively ${ }^{6}$. The mPTP opening detected by calcein quenching rate was reduced by oligomycin and DCCD as efficiently as by $\operatorname{CsA}^{18}$. In addition, small molecules mimicking the southern moiety of oligomycin target the $c$ subunit(s) of $F_{O}$ domain and inhibit the mPTP without affecting ATP synthesis ${ }^{19}$. $\mathrm{Ca}^{2+}$ binding to the $\mathrm{F}_{1}$ domain involves a catalytic mechanism that may be cation-dependent ${ }^{6}$. The higher $\mathrm{Ca}^{2+}$ steric hindrance with respect to $\mathrm{Mg}^{2+}$ (Fig. 3 ) implies the onset a more flexible coordination geometry characterized by irregular bond distances and angles in the enzyme catalytic sites of $B$ subunit. The geometry of the cofactor-binding site of the globular $(\alpha B)_{3}$ structure of $F_{1}$ domain can change and would be transmitted to the nearby OSCP subunit and through the peripheral stalk reach the membrane where the $\mathrm{mPTP}_{\text {opens }} \mathrm{7}^{720}$. To this end, the aim is to discover new drugs that selectively prevent the $\mathrm{Ca}^{2+}$ binding in the catalytic sites of $\mathrm{F}_{1}$ domain thus preventing the MPTP formation.

To sum up, the discovery of molecular identity and mechanism of mPTP phenomenon will significantly improve the search for new mitochondrial drugs that will have an effective pharmaceutical solution in the treatment of many diseases by preventing the formation of the channel with selective mPTP inhibitors. Conversely, the extreme refractoriness to death of cancer cells suggests that these cells have evolved mechanisms to escape mPTP opening by desensitizing the MPTP to mPTP regulators. Since the decreased propensity to mPTP opening favours tumour progression, the MPTP re-activation arises as promising strategy to circumvent oncogenic defects.

\section{References}

1. Bernardi P, Rasola A, Forte M, Lippe G. The Mitochondrial Permeability Transition Pore: Channel Formation by F-ATP Synthase, Integration in Signal Transduction, and Role in Pathophysiology. Physiol Rev 2015;95(4):1111-1155.

2. Izzo V, Bravo-San Pedro JM, Sica V, Kroemer G, Galluzzi L. Mitochondrial Permeability Transition: New Findings and Persisting Uncertainties. Trends Cell Biol 2016;26(9):655-667.

3. Biasutto L, Azzolini M, Szabò I, Zoratti M. The mitochondrial permeability transition pore in AD 2016: An update. Biochim Biophys Acta 2016;1863(10):2515-2530.

4. Giorgio V, von Stockum S, Antoniel M, Fabbro A, Fogolari F, Forte M, Glick GD, Petronilli V, Zoratti M, Szabó I, Lippe G, Bernardi P. Dimers of mitochondrial ATP synthase form the permeability transition pore. Proc Natl Acad Sci USA 2013;110(15):5887-5892.

5. Bonora M, Bononi A, De Marchi E, Giorgi C, Lebiedzinska M, Marchi S, Patergnani S, Rimessi A, Suski JM, Wojtala A, Wieckowski MR, Kroemer G, Galluzzi L, Pinton P. Role of the c subunit of the FO ATP synthase in mitochondrial permeability transition. Cell Cycle 2013;12(4):674-683. 
6. Nesci S, Trombetti F, Ventrella V, Pirini M, Pagliarani A. Kinetic properties of the mitochondrial F1FOATPase activity elicited by $\mathrm{Ca}(2+)$ in replacement of $\mathrm{Mg}(2+)$. Biochimie 2017;14073-81.

7. Giorgio V, Burchell V, Schiavone M, Bassot C, Minervini G, Petronilli V, Argenton F, Forte M, Tosatto S, Lippe G, Bernardi P. $\mathrm{Ca}(2+)$ binding to F-ATP synthase $\beta$ subunit triggers the mitochondrial permeability transition. EMBO Rep 2017;18(7):1065-1076.

8. Nesci S. A Lethal Channel between the ATP Synthase Monomers. Trends Biochem Sci 2018;43(5):311313.

9. Alavian KN, Beutner G, Lazrove E, Sacchetti S, Park H-A, Licznerski P, Li H, Nabili P, Hockensmith K, Graham M, Porter GA, Jonas EA. An uncoupling channel within the c-subunit ring of the F1FO ATP synthase is the mitochondrial permeability transition pore. Proc Natl Acad Sci USA 2014;111(29):10580-10585.

10. Neginskaya MA, Solesio ME, Berezhnaya EV, Amodeo GF, Mnatsakanyan N, Jonas EA, Pavlov EV. ATP Synthase C-Subunit-Deficient Mitochondria Have a Small Cyclosporine A-Sensitive Channel, but Lack the Permeability Transition Pore. Cell Rep 2019;26(1):11-17.e2.

11. Campo G, Morciano G, Pavasini R, Bonora M, Sbano L, Biscaglia S, Bovolenta M, Pinotti M, Punzetti S, Rizzo P, Aquila G, Giorgi C, Ferrari R, Pinton P. Fo ATP synthase $C$ subunit serum levels in patients with ST-segment Elevation Myocardial Infarction: Preliminary findings. Int J Cardiol 2016;221993-997.

12. Ludtmann MHR, Angelova PR, Horrocks MH, Choi ML, Rodrigues M, Baev AY, Berezhnov AV, Yao Z, Little D, Banushi B, Al-Menhali AS, Ranasinghe RT, Whiten DR, Yapom R, Dolt KS, Devine MJ, Gissen P, Kunath T, Jaganjac M, Pavlov EV, Klenerman D, Abramov AY, Gandhi S. $\alpha$-synuclein oligomers interact with ATP synthase and open the permeability transition pore in Parkinson's disease. Nat Commun 2018;9(1):2293.

13. Giorgio V, Bisetto E, Soriano ME, Dabbeni-Sala F, Basso E, Petronilli V, Forte MA, Bernardi P, Lippe G. Cyclophilin D modulates mitochondrial FOF1-ATP synthase by interacting with the lateral stalk of the complex. J Biol Chem 2009;284(49):33982-33988.

14. Nesci S, Ventrella V, Trombetti F, Pirini M, Pagliarani A. Preferential nitrite inhibition of the mitochondrial F1FO-ATPase activities when activated by $\mathrm{Ca}(2+)$ in replacement of the natural cofactor Mg(2+). Biochim Biophys Acta 2016;1860(2):345-353.

15. Baines CP, Kaiser RA, Purcell NH, Blair NS, Osinska H, Hambleton MA, Brunskill EW, Sayen MR, Gottlieb RA, Dorn GW, Robbins J, Molkentin JD. Loss of cyclophilin D reveals a critical role for mitochondrial permeability transition in cell death. Nature 2005;434(7033):658-662.

16. Sileikyte J, Roy S, Porubsky P, Neuenswander B, Wang J, Hedrick M, Pinkerton AB, Salaniwal S, Kung P, Mangravita-Novo A, Smith LH, Bourdette DN, Jackson MR, Aubé J, Chung TDY, Schoenen FJ, Forte MA, Bernardi P. Small Molecules Targeting the Mitochondrial Permeability Transition. In: Probe Reports from the NIH Molecular Libraries Program. Bethesda (MD): National Center for Biotechnology Information (US).;

17. Clarke SJ, McStay GP, Halestrap AP. Sanglifehrin A acts as a potent inhibitor of the mitochondrial permeability transition and reperfusion injury of the heart by binding to cyclophilin- $D$ at a different site from cyclosporin A. J Biol Chem 2002;277(38):34793-34799.

18. Bonora M, Morganti C, Morciano G, Pedriali G, Lebiedzinska-Arciszewska M, Aquila G, Giorgi C, Rizzo P, Campo G, Ferrari R, Kroemer G, Wieckowski MR, Galluzzi L, Pinton P. Mitochondrial permeability 
transition involves dissociation of F1FO ATP synthase dimers and C-ring conformation. EMBO Rep 2017;18(7):1077-1089.

19. Morciano G, Preti D, Pedriali G, Aquila G, Missiroli S, Fantinati A, Caroccia N, Pacifico S, Bonora M, Talarico A, Morganti C, Rizzo P, Ferrari R, Wieckowski MR, Campo G, Giorgi C, Trapella C, Pinton P. Discovery of Novel 1,3,8-Triazaspiro[4.5]decane Derivatives That Target the c Subunit of F1/FOAdenosine Triphosphate (ATP) Synthase for the Treatment of Reperfusion Damage in Myocardial Infarction. J Med Chem 2018;61(16):7131-7143.

20. Nesci $\mathrm{S}$. Mitochondrial permeability transition, $\mathrm{F}_{1} \mathrm{~F}_{\mathrm{O}}-\mathrm{ATPase}$ and calcium: an enigmatic triangle. EMBO Rep 2017;18(8):1265-1267.

\section{Figure Legends}

Figure 1. $F_{1} F_{0}$-ATPase monomer in the inner mitochondrial membrane. Protein subunits are drawn as ribbon representations (modified PDB ID codes: 5ARA and 6B2Z). Olive, $\alpha$ subunits; red, $b$ subunits; blue, $\gamma$ subunit; fuchsia, $\delta$ subunit; turquoise, $\varepsilon$ subunit; orange, ring of $c$ subunits; violet, $a$ subunit; purple, A6L subunit; gold, $f$ subunit; green, $b$ subunit; salmon, $d$ subunit; sky-blue, F6 subunit; grey, OSCP subunit. $e$ and $g$ subunits drawn in ball and stick mode, are blue and light blue, respectively; pink, $6.8 \mathrm{kDa}$ proteolipid (i/j subunit in yeast); brown, DAPIT ( $k$ subunit in yeast) is truncated.

Figure 2. Putative involvement of the $\mathrm{F}_{1} \mathrm{~F}_{0}$-ATPase complexes in $\mathrm{MPTP}$ formation by switching between $\mathrm{Mg}^{2+}$ activated and $\mathrm{Ca}^{2+}$-activated $\mathrm{F}_{1} \mathrm{~F}_{\mathrm{O}}$-ATPase. Protein subunits are drawn as ribbon representations (modified PDB ID codes: 5ARA and 6B2Z). The colours of the subunits are the same as in Fig. 1. ATP synthesis and hydrolysis are physiological features of the $\mathrm{Mg}^{2+}$-activated $\mathrm{F}_{1} \mathrm{~F}_{0}$-ATPase (on the left), while only ATP hydrolysis can be supported by the $\mathrm{Ca}^{2+}$-activated $\mathrm{F}_{1} \mathrm{~F}_{\mathrm{O}}$-ATPase (on the right) which triggers the mPTP opening. The bidirectional red arrow depicts the presumed region of MPTP opening.

Figure 3. Putative arrangement of $\mathrm{Mg}^{2+}$ and $\mathrm{Ca}^{2+}$ within key aminoacid residues in the catalytic 8 subunit of the $\mathrm{F}_{1} \mathrm{~F}_{\mathrm{O}^{-}}$-ATPase. A) MgATP ( $\mathrm{Mg}^{2+}$ as turquoise sphere and ATP in stick mode) and $\mathrm{B}$ ) CaATP $\left(\mathrm{Ca}^{2+}\right.$ as grey sphere and ATP as stick mode). The electrondensity map of $\mathrm{Mg}^{2+}$ and $\mathrm{Ca}^{2+}$ is drawn as a sphere. The divalent cations are directly coordinated by Thr 163 and the oxygen atoms of $\beta$ and $\gamma$-phosphates of ATP, while Arg 189, Asp 256 and Glu 192 are indirectly coordinated by three ordered water molecules.

\section{Bio-sketch}

His research is focused on the mitochondrial bioenergetics. In particular, his interest is addressed to the study of enzymatic catalysis of mitochondrial ATP synthase due to post-translational chemical modifications and the physiological role of the enzyme activated by calcium.

\section{https://www.unibo.it/sitoweb/salvatore.nesci/en}




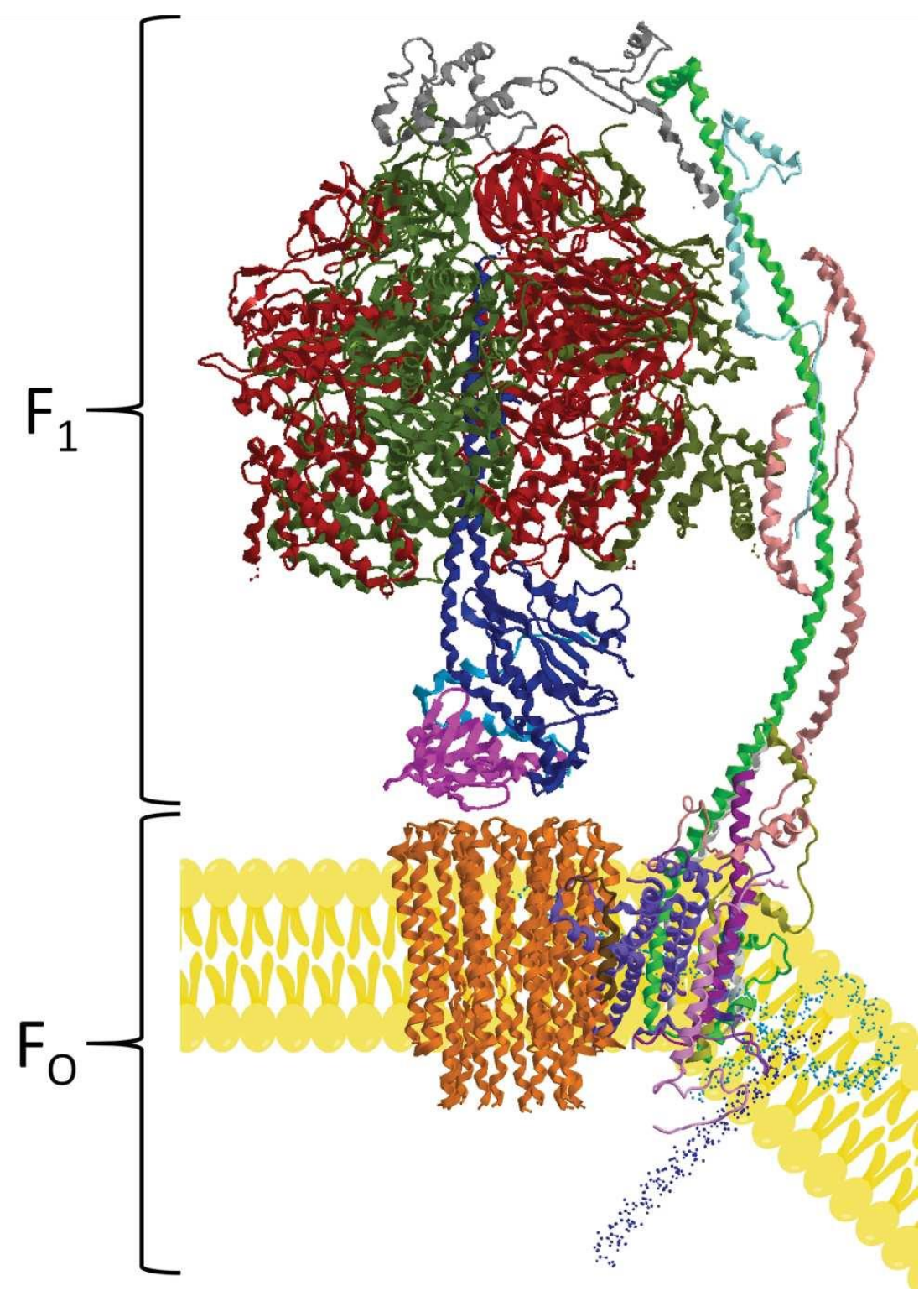

Figure 1. F1FO-ATPase monomer in the inner mitochondrial membrane. Protein subunits are drawn as ribbon representations (modified PDB ID codes: 5ARA and 6B2Z). Olive, a subunits; red, $\beta$ subunits; blue, $Y$ subunit; fuchsia, $\delta$ subunit; turquoise, $\varepsilon$ subunit; orange, ring of c subunits; violet, a subunit; purple, A6L subunit; gold, f subunit; green, b subunit; salmon, d subunit; sky-blue, F6 subunit; grey, OSCP subunit. e and $\mathrm{g}$ subunits drawn in ball and stick mode, are blue and light blue, respectively; pink, $6.8 \mathrm{kDa}$ proteolipid ( $\mathrm{i} / \mathrm{j}$ subunit in yeast); brown, DAPIT ( $\mathrm{k}$ subunit in yeast) is truncated. 


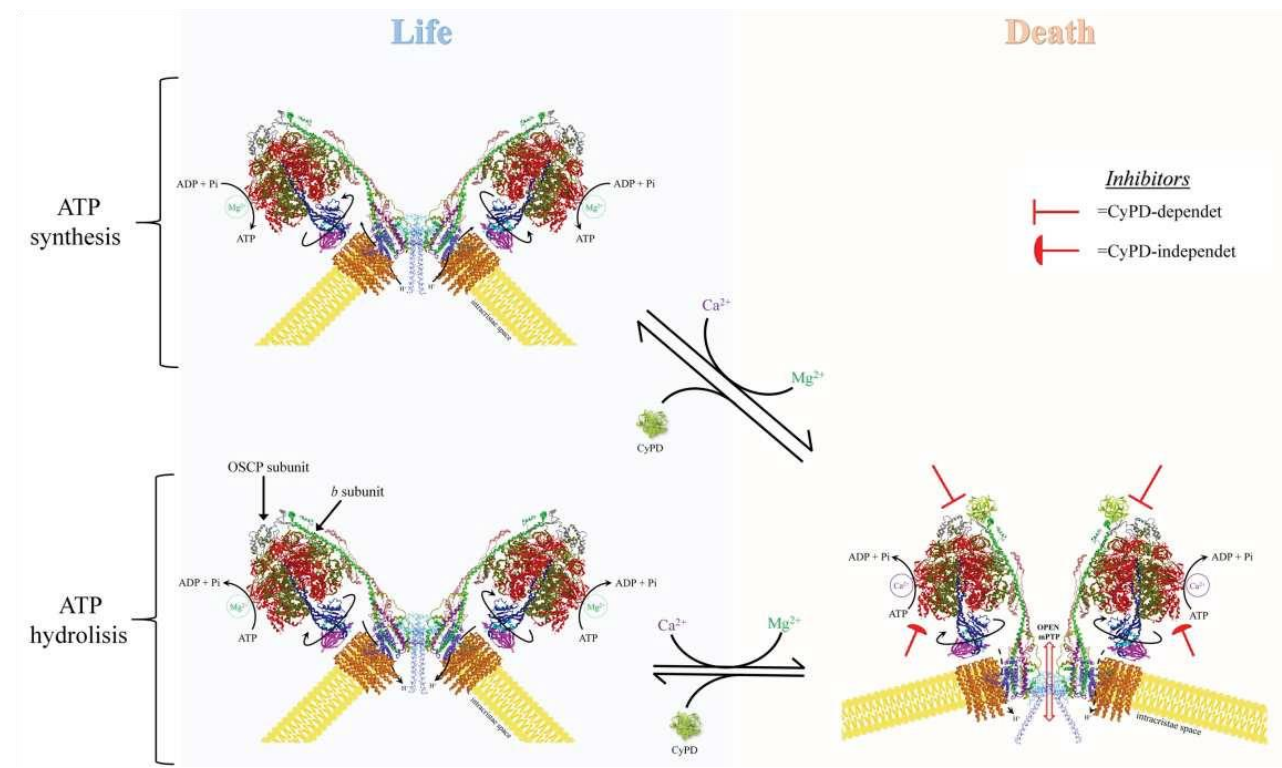

Figure 2. Putative involvement of the F1FO-ATPase complexes in MPTP formation by switching between $\mathrm{Mg} 2+$-activated and Ca2+-activated F1FO-ATPase. Protein subunits are drawn as ribbon representations (modified PDB ID codes: 5ARA and 6B2Z). The colours of the subunits are the same as in Fig. 1. ATP synthesis and hydrolysis are physiological features of the Mg2+-activated F1FO-ATPase (on the left), while only ATP hydrolysis can be supported by the Ca2+-activated F1FO-ATPase (on the right) which triggers the mPTP opening. The bidirectional red arrow depicts the presumed region of MPTP opening.

$107 \times 64 \mathrm{~mm}(600 \times 600 \mathrm{DPI})$ 
A)

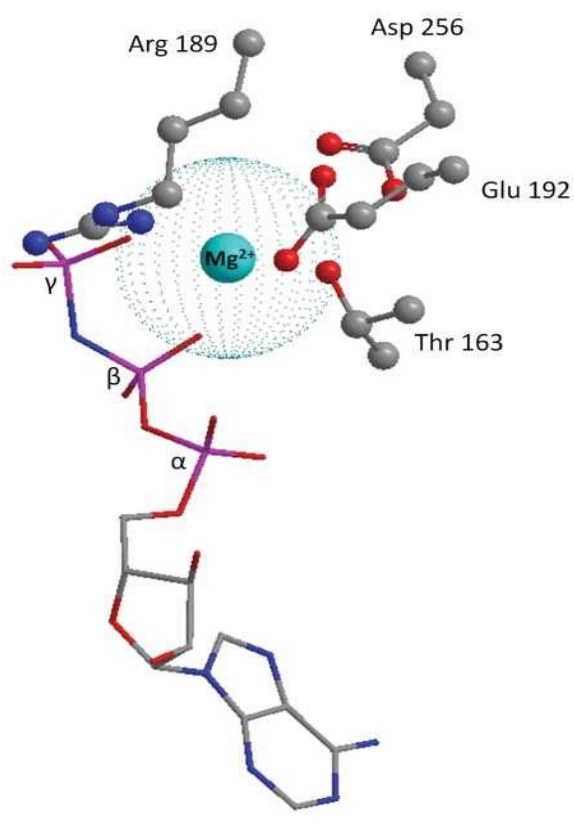

B)

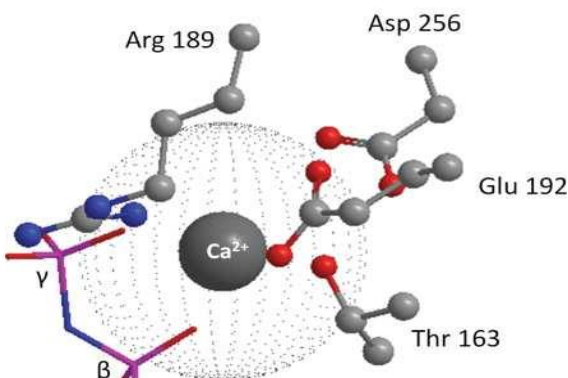

Figure 3. Putative arrangement of $\mathrm{Mg} 2+$ and $\mathrm{Ca} 2+$ within key aminoacid residues in the catalytic $\beta$ subunit of the F1FO-ATPase. A) MgATP (Mg2+ as turquoise sphere and ATP in stick mode) and B) CaATP (Ca2+ as grey sphere and ATP as stick mode). The electrondensity map of $\mathrm{Mg} 2+$ and $\mathrm{Ca} 2+$ is drawn as a sphere. The divalent cations are directly coordinated by Thr 163 and the oxygen atoms of $\beta$ and $y$-phosphates of ATP, while Arg 189, Asp 256 and Glu 192 are indirectly coordinated by three ordered water molecules. 